\title{
Desafios no cumprimento do calendário vacinal de crianças de 0 a 5 anos: uma
} revisão integrativa

\author{
Challenges in compliance with the vaccine calendar for children from 0 to 5 years: an integrative \\ review
}

Retos en cumplimiento del calendario de vacunas para niños de 0 hasta 5 años: una revisión integradora

\author{
Albanir Crislys Pereira da Silva \\ ORCID: https://orcid.org/0000-0001-7063-9190 \\ Instituto de Medicina Integral Prof. Fernando Figueira, Brasil \\ E-mail: albanircris@gmail.com \\ Emanuela Batista Ferreira e Pereira \\ ORCID: https://orcid.org/0000-0003-4665-4379 \\ Universidade de Pernambuco, Brasil \\ E-mail: emanuela.pereira@upe.br \\ Claudia Fabiana Lucena Spíndola \\ ORCID: https://orcid.org/0000-0001-5996-3846 \\ Prefeitura da Cidade do Recife, Brasil \\ E-mail: claudiaflspindola@gmail.com \\ Priscila Diniz de Carvalho Martins \\ ORCID: https://orcid.org/0000-0002-7794-2130 \\ Prefeitura da Cidade do Recife, Brasil \\ E-mail: prifermeira@hotmail.com \\ Virginian Cristiana Amorim da Silva \\ ORCID: https://orcid.org/0000-0002-5996-4133 \\ Instituto Pernambucano de Ensino Superior, Brasil \\ E-mail: viamorim2018@gmail.com \\ Giselda Bezerra Correia Neves \\ ORCID: https://orcid.org/0000-0002-7902-5184 \\ Hospital da Restauração Gov. Paulo Guerra, Brasil \\ E-mail: giseldabcneves@gmail.com \\ Geyse Tavares de Souza \\ ORCID: https://orcid.org/0000-0001-5209-3710 \\ Universidade de Pernambuco, Brasil \\ E-mail: geyse.souza@upe.br \\ Keilla Isabelle da Silva Santos \\ ORCID: https://orcid.org/0000-0002-5723-9333 \\ Instituto Pernambucano de Ensino Superior, Brasil \\ E-mail: enfer.keillaisa@gmail.com \\ Rayssa Sydnara Angelo Tavares \\ ORCID: https://orcid.org/0000-0001-7179-0116 \\ Instituto Pernambucano de Ensino Superior, Brasil \\ E-mail: rayssa_tavares222@hotmail.com \\ Stefane Monte da Silva Macedo \\ ORCID: https://orcid.org/0000-0002-0973-8509 \\ Instituto Pernambucano de Ensino Superior, Brasil \\ E-mail: s_monte29@hotmail.com \\ Márcia Andréa Albuquerque Santos de Mendonça \\ ORCID: https://orcid.org/0000-0002-9368-9278 \\ Universidade de Pernambuco, Brasil \\ E-mail: marcia.mendonca@upe.br \\ Elisabeth Lima Dias da Cruz \\ ORCID: https://orcid.org/0000-0003-1763-6956 \\ Universidade de Pernambuco, Brasil \\ E-mail: elisabeth.cruz@upe.br
}

\section{Resumo}

Este artigo tem como objetivo compreender os principais desafios que levam os pais/responsáveis de crianças de 0 a 5 anos ao não cumprimento do calendário vacinal e seus fatores associados. Trata-se de uma revisão integrativa de literatura nas bases de dados do LILACS, Biblioteca Virtual de Saúde / BIREME, Scielo, MEDLINE e Base de dados 
de enfermagem. Foi utilizado os descritores de saúde: vacinas, cobertura vacinal, crianças e saúde pública, aplicando o operador boleano “AND”. Após aplicar os critérios de inclusão e exclusão, localizou-se 394 artigos, consistindo numa amostra final de 10 artigos. Diante dos resultados os motivos para os pais e/ou responsáveis não realizarem as vacinas das crianças de 0 a 5 anos foram: julgar as vacinas como sendo desnecessárias, medo dos efeitos colaterais e optar por estilos de vida naturais. Além da existência dos fatores que interferem nesse processo como a ausência de imunobiológicos e a cultura familiar os principais. Dessa maneira, os pais e/ou responsáveis que abordaram achar desnecessárias as vacinas, ter dúvidas sobre sua eficácia, se apegam a fontes incompleta de informação, sendo isso uma barreira a ser solucionada. Por outro lado, o serviço público de saúde precisa melhorar, como no fornecimento de imunobiológicos para os serviços de atenção básica, fazendo com que os estoques se mantenham abastecidos para não haver falta dos mesmos.

Palavras-chave: Vacinas; Cobertura vacinal; Crianças; Saúde pública.

\begin{abstract}
This article aims to understand the main challenges that lead parents/guardians of children aged 0 to 5 years to not comply with the vaccination schedule and its associated factors. This is an integrative literature review in LILACS databases, Virtual Health Library / BIREME, Scielo, MEDLINE and Nursing database. The following health descriptors were used: vaccines, vaccination coverage, children and public health, applying the Boolean operator "AND". After applying the inclusion and exclusion criteria, 394 articles were located, consisting of a final sample of 10 articles. In view of the results, the reasons for parents and/or guardians not to vaccinate children aged 0 to 5 years were: judging the vaccines as unnecessary, fear of side effects and opting for natural lifestyles. In addition to the existence of factors that interfere in this process, such as the absence of immunobiologicals and family culture, the main ones. In this way, parents and/or guardians who mentioned that they thought the vaccines were unnecessary, had doubts about their effectiveness, clung to incomplete sources of information, which was a barrier to be resolved. On the other hand, the public health service needs to improve, as in the provision of immunobiologicals for primary care services, ensuring that stocks remain stocked so there is no shortage of them.
\end{abstract}

Keywords: Vaccines; Vaccine coverage; Kids; Public health.

\title{
Resumen
}

Este artículo tiene como objetivo comprender los principales desafíos que llevan a los padres / tutores de niños de 0 a 5 años a no cumplir con el calendario de vacunación y sus factores asociados. Se trata de una revisión bibliográfica integradora en las bases de datos LILACS, Biblioteca Virtual en Salud / BIREME, Scielo, MEDLINE y base de datos de Enfermería. Se utilizaron los siguientes descriptores de salud: vacunas, coberturas de vacunación, niños y salud pública, aplicando el operador booleano "Y". Tras aplicar los criterios de inclusión y exclusión, se localizaron 394 artículos, constituidos por una muestra final de 10 artículos. A partir de los resultados, las razones por las que los padres y / o tutores no vacunaron a los niños de 0 a 5 años fueron: juzgar las vacunas como innecesarias, miedo a los efectos secundarios y optar por estilos de vida naturales. Además de la existencia de factores que interfieren en este proceso, como la ausencia de inmunobiológicos y cultura familiar, los principales. De esta forma, los padres y / o tutores que mencionaron que pensaban que las vacunas eran innecesarias, tenían dudas sobre su efectividad, se aferraban a fuentes de información incompletas, lo cual era una barrera por resolver. Por otro lado, el servicio de salud pública debe mejorar, como en la provisión de inmunobiológicos para los servicios de atención primaria, asegurando que las existencias permanezcan almacenadas para que no haya escasez de ellas.

Palabras clave: Vacunas; Cobertura de la vacuna; Niños; Salud pública.

\section{Introdução}

O Programa Nacional de Imunizações (PNI) foi formulado em 1973 sob determinação do Ministério da Saúde (MS) com o objetivo de coordenar às ações de imunização no país, que são desenvolvidas rotineiramente na rede de serviços públicos ou privados, tendo em vista a obrigatoriedade do cumprimento do calendário vacinal (Ferreira et al, 2018).

Surgiu em 1976 a partir de uma experiência realiza pelo médico inglês Edward Jenner a famosa "vacina", a primeira doença combatida através da vacinação foi a varíola, apesar de tantos anos de descoberta ainda é utilizada como o principal meio de prevenção de doenças infecciosas que podem levar a morte, a mesma chegou ao Brasil em 1804, pelo Marquês Barbacena (Repa, 2018).

De acordo com Repa (2018) “as vacinas objetivam imunizar os pacientes fazendo com que seu sistema imunológico trabalhe em favor deste, prevenindo contra uma série de doenças”, ainda existem doenças que não são prevenidas através da vacina por elas estarem em estudos ou pelo seu alto grau de letalidade.

A cobertura vacinal não atinge $100 \%$ do seu percentual, isso é um grande desafio para a saúde pública, a própria 
Organização Mundial de Saúde (OMS) reconhece e recomenda que ocorra ao menos 95\% de cobertura sendo um considerável índice para o controle e erradicação de doenças imunopreveníveis (Braz et al., 2016).

A BCG é o imunobiológico com menores taxas de atraso, muitos municípios dispõem da mesma em estabelecimentos de parto, pois é recomendada a administração imediata logo após o nascimento (Clara et al., 2020), em contrapartida a meningocócica $\mathrm{C}$ e a pneumocócica 10 valente tem um elevado número de atraso (Figueiras et al., 2018), essas vacinas foram implantadas no calendário vacinal das crianças no ano de 2010 e a partir daí aumentou o percentual de vacinas incompletas (Silva et al., 2018).

Nos anos que há implantação de novas vacinas há uma pequena queda nos resultados esperados, segundo o mesmo estudo observou-se que após os 6 meses ocorre um declínio no cumprimento do calendário, pois completa o esquema de várias vacinas (Ferreira et al., 2018).

Mediante o cenário existente no século XXI, onde as pessoas estão dotadas de conhecimentos e tecnologias, ainda existem pais que optam por não imunizar seus filhos por uma série de fatores, dentre eles: as reações adversas, não garantir a proteção, optam pelo estilo de vida natural, fatores filosóficos e religiosos (Barbieri et al., 2017)

Levando em consideração o conhecimento desses pais acerca da lei e dos pontos positivos e negativos de não vacinar, seu maior temor é de enfrentar o tribunal, em vez de lidar com as consequências da (não) vacinação (Barbieri \& Couto \& Aith, 2017). Chama atenção para os fatores identificados em presente situação no País, onde crianças de classe social mais alta têm a ausência de registro de vacinas novas introduzidas (Silva et al., 2018).

Em estudo realizado em uma escola de medicina privada em São Paulo, no ano de 2016, observou-se que 43,4\% dos estudantes entrevistados e 41,0\% dos médicos conhecem alguém que se recuse ao ato de vacinar seus filhos, 54,7\% e 59,0\% conheciam pessoas que se recusam a receber vacinas, $43,4 \%$ e 56,4\% acham razoável respeitar a vontade do indivíduo, mas que seria necessário explicar a consequência desse ato ao paciente, uma vez que a recusa vacinal compromete a saúde de todos (Mizuta et al., 2018).

O cenário de movimentos anti vacinais voltou a acontecer, a mídia é a principal responsável, por conta das variedades de notícias muitas vezes falsas, chega a ser de suma indignação ter abordado que as vacinas causavam o autismo levando outra visão à população, sem nenhuma comprovação cientifica (Medeiros, 2019).

Considerando a queda nos números da vacinação em crianças de 0 a 5 anos e o aumento em casos de doenças transmissíveis que já haviam sido erradicadas, é necessária uma análise junto às mães para entender os principais motivos que as levam a deixar de vacinar seus filhos, conforme o calendário do PNI, mesmo existindo o Decreto 78.231/76, no seu capítulo 2 e $\operatorname{art}^{\circ} 29$, que diz: "é dever de todo cidadão submeter-se e os menores dos quais tenha a guarda ou responsabilidade, à vacinação obrigatória" (Civil, 2020).

Desta forma, esta pesquisa se justifica pela importância para a saúde pública e saúde da população. A cobertura vacinal é um dos assuntos de maior repercussão, não só do Brasil, mas também de vários outros países. No qual será analisado a cobertura vacinal de um determinado território, como também, a importância que a mãe dá a está atitude, que irá contribuir ou não, para que o ato de vacinar os menores seja executado. Uma vez identificando o motivo da não vacinação por algumas famílias poderá ser trabalhado na resolutividade do problema identificado.

Esta pesquisa tem como objetivo compreender os principais desafios que levam os pais/responsáveis de crianças de 0 a 5 anos ao não cumprimento do calendário vacinal e seus fatores associados.

\section{Metodologia}

Este trabalho trata-se de uma Revisão Integrativa da Literatura, cuja abordagem metodológica é mais ampla referente as demais revisões, permitindo compreender por completo os fenômenos analisados, dentro de um conceito complexo de teoria 
e problemas de saúde relevantes para a enfermagem (Soares et al., 2010).

Foi realizada uma busca nas bases de dados do LILACS (Literatura Latino-Americana e do Caribe em Ciências da Saúde), na Biblioteca Virtual de Saúde (BVS/BIREME), na biblioteca do Scielo, e nas bases do Medline (Sistema Online de Busca e Análise de Literatura Médica) e na Base de dados de enfermagem (Bdenf), a partir do cruzamento dos descritores de saúde (DECS): vacinas, cobertura vacinal, crianças e saúde pública. Para realização do cruzamento dos descritores de saúde será utilizado o operador booleano "AND".

Foram utilizados como critérios de inclusão: artigos disponíveis na íntegra, no idioma português e inglês entre o período de publicação de 2015 a 2020, artigos que tenha como população pais e/ou responsáveis. Os critérios de exclusão foram: artigos duplicados nas bases de dados; artigos de revisão; artigos que contemplem o conteúdo de calendário vacinal para o público com idade superior a 5 anos; população do estudo não seja os pais e/ou responsáveis e que não apresentem fatores associados.

Para coleta dos dados foi elaborado um instrumento, no qual foi submetido a validação aparente e de conteúdo aos professores que acompanham o andamento da pesquisa. $\mathrm{O}$ instrumento contempla os seguintes itens: identificação do artigo original, características metodológicas do estudo, população alvo, conhecimento sobre calendário vacinal, fatores associados que interferem na vacinação de crianças de 0 a 5 anos.

Demanda uma abordagem organizada, levando em consideração as características, estruturas e o tipo de cada artigo (experimental, laboratorial, documental, bibliográfico ou revisão e estudo de caso) incluso na pesquisa. Iniciou-se com uma leitura prévia dos artigos que foram separados, observando a experiência clínica do pesquisador que contribuiu na validação dos métodos e resultados, selecionando os pontos a serem utilizados na prática, portanto, para que isso seja contemplado, é necessário ter uma visão crítica para analisar cada artigo. Os artigos resultantes foram lidos na íntegra e em seguida analisados através da metodologia PRISMA (Figura 1). 
Figura 1: Fluxograma e critérios de seleção e inclusão dos artigos.

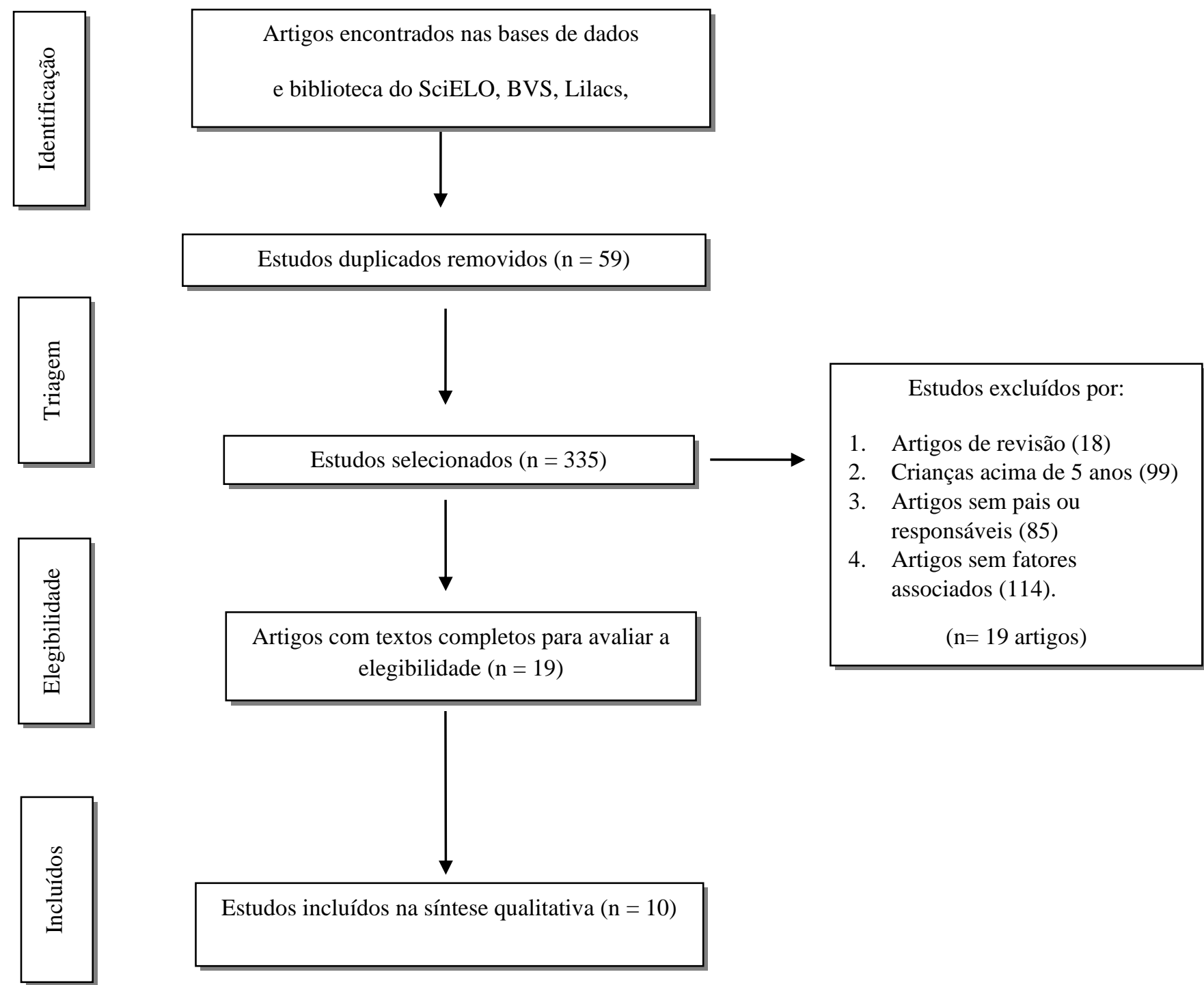

Fonte: "Prisma: Transparent Reporting of Systematic Reviews and Meta-Analyses".

\section{Resultados}

Diante dessa pesquisa realizada nas bases de dados, referente ao tema, foram inclusos 10 artigos para análise final, logo após foi construído uma tabela com dados referente aos artigos, apresentando a identificação do artigo original, País, população, conhecimento dos pais e/ou responsáveis sobre o calendário vacinal, motivos de não realizar da vacina e fatores associados que interferem na vacinação de crianças de 0 a 5 anos. 
Tabela 1 - Desafios encontrados pelos pais e/ou responsáveis para o não cumprimento do calendário vacinal de crianças de 0 a 5 anos.

\begin{tabular}{|c|c|c|c|c|c|}
\hline Autores/ Ano & País & População & $\begin{array}{c}\text { Conhecimento dos pais } \\
\text { e/ou responsáveis }\end{array}$ & $\begin{array}{l}\text { Motivos de não realizar a } \\
\text { vacina }\end{array}$ & $\begin{array}{l}\text { Fatores associados que } \\
\text { interferem na vacinação }\end{array}$ \\
\hline Horne et al., 2015 & Estados Unidos & $\begin{array}{l}\text { Homens e mulheres; } \\
\text { Idade média de idade entre } \\
35 \text { e } 40 \text { anos }\end{array}$ & Nível intermediário & $\begin{array}{l}\text { - Julga algumas vacinas } \\
\text { como desnecessárias }\end{array}$ & $\begin{array}{l}\text { - } \\
\text { Incertezas quanto a } \\
\text { eficácia das vacinas; } \\
\text { - Insegurança em relação } \\
\text { a vacina contra a } \\
\text { influenza. }\end{array}$ \\
\hline Barros et al., 2015 & Brasil & $\begin{array}{l}\text { Técnicos ou auxiliares de } \\
\text { enfermagem; pais e/ou } \\
\text { responsáveis }\end{array}$ & Baixo nível & $\begin{array}{l}\text { - } \\
\text { - } \text { Criança doente; } \\
\text { - } \text { Falta de água } \\
\text { - } \text { Falta de tempo; } \\
\text { - Esquecimento; } \\
\text { - Perda da caderneta da } \\
\text { criança. }\end{array}$ & 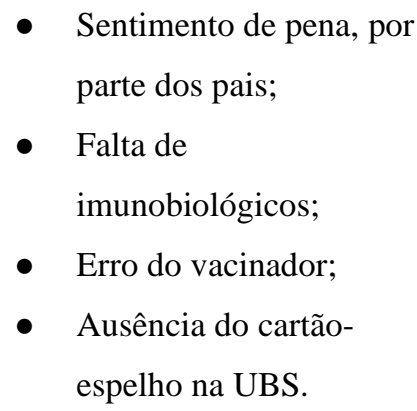 \\
\hline Braz et al, 2016 & Estados Unidos & $\begin{array}{l}\text { Pais e/ou responsáveis e } \\
\text { funcionários de creches }\end{array}$ & Alto nível & $\begin{array}{l}\text { O valor das vacinas; } \\
\text { O fato das vacinas } \\
\text { serem pagas no EUA. }\end{array}$ & ---------- \\
\hline
\end{tabular}


Research, Society and Development, v. 10, n. 14, e531101422343, 2021

(CC BY 4.0) | ISSN 2525-3409 | DOI: http://dx.doi.org/10.33448/rsd-v10i14.22343

\begin{tabular}{|c|c|c|c|c|c|c|}
\hline Barbieri, Couto, Aith, 2017 & Brasil & $\begin{array}{l}\text { Pais e/ou responsáveis com } \\
\text { filho de até } 5 \text { anos de idade }\end{array}$ & Alto nível & $\bullet$ & $\begin{array}{l}\text { Erradicação da doença } \\
\text { ou doença leve; } \\
\text { Medo dos eventos } \\
\text { adversos; } \\
\text { Crítica à composição } \\
\text { das vacinas, sua } \\
\text { eficácia; } \\
\text { Julga o calendário de } \\
\text { vacinação do país, ao } \\
\text { interesse financeiro e } \\
\text { lucro das indústrias } \\
\text { farmacêuticas; } \\
\text { Escolhe outras formas } \\
\text { de proteção à saúde } \\
\text { (como estilo de vida } \\
\text { mais natural). }\end{array}$ & $\begin{array}{l}\text { - Houve crítica a VOP e } \\
\text { a Tríplice viral pelo } \\
\text { risco de eventos } \\
\text { adversos } \\
\text { - } \\
\text { Rotavírus e gripe, por } \\
\text { considerarem doenças } \\
\text { leves. }\end{array}$ \\
\hline Xi Li et al., 2017 & Kiribati & $\begin{array}{l}\text { Mulheres grávidas, } \\
\text { trabalhadores de saúde e } \\
\text { agentes de saúde }\end{array}$ & Baixo nível & $\bullet$ & $\begin{array}{l}\text { Mais de } 50 \% \text { das } \\
\text { crianças nasceram em } \\
\text { casa; } \\
\text { Profissionais de saúde } \\
\text { não levaram a vacina } \\
\text { contra hepatite B para } \\
\text { partos domiciliares. }\end{array}$ & $\begin{array}{l}\text { Falta de conhecimento } \\
\text { sobre os fatores de risco } \\
\text { do vírus da hepatite B }\end{array}$ \\
\hline
\end{tabular}


Research, Society and Development, v. 10, n. 14, e531101422343, 2021

(CC BY 4.0) | ISSN 2525-3409 | DOI: http://dx.doi.org/10.33448/rsd-v10i14.22343

\begin{tabular}{|c|c|c|c|c|c|}
\hline Dubé et al., 2017 & Canadá & $\begin{array}{l}\text { Pais e/ou responsáveis com } \\
\text { pelo menos uma criança de } \\
24 \text { a } 59 \text { meses de idade }\end{array}$ & Nível intermediário & 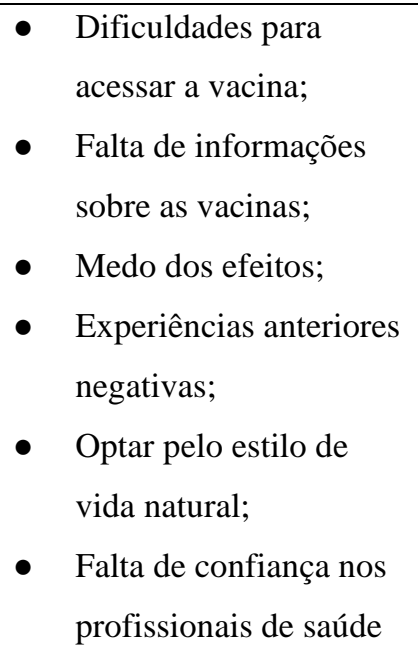 & $\begin{array}{ll}\text { - } & \text { Práticas religiosas ou } \\
& \text { espirituais; } \\
\text { - } & \text { Não reconhecer seu } \\
& \text { papel como pai; } \\
\text { - } & \text { Buscar informações na } \\
\text { internet; } \\
\text { - } \quad \text { Baixa intenção de } \\
\text { vacinar seus filhos }\end{array}$ \\
\hline Nzioki et al., 2017 & Quênia & $\begin{array}{l}\text { Mulheres com um filho de } \\
9-12 \text { meses }\end{array}$ & Nível intermediário & - Fatores religiosos; & $\begin{array}{l}\text { - Falta de rastreamento } \\
\text { por parte da Unidades }\end{array}$ \\
\hline Biezen et al., 2018 & Austrália & $\begin{array}{l}\text { Pais e/ou responsáveis de } \\
\text { crianças }<5 \text { anos }\end{array}$ & Nível intermediário & $\begin{array}{l}\text { - } \text { Falta de tempo para } \\
\text { - } \text { O cuscutir sobre a vacina; } \\
\text { - } \\
\text { - } \\
\text { Publicidade nacina; } \\
\text { criança comativa; } \\
\text { agulhas }\end{array}$ & $\begin{array}{l}\text { - Possuem incertezas } \\
\text { quanto a eficácia; } \\
\text { - Insegurança à vacina } \\
\text { contra influenza e sua } \\
\text { eficácia }\end{array}$ \\
\hline
\end{tabular}


Research, Society and Development, v. 10, n. 14, e531101422343, 2021

(CC BY 4.0) | ISSN 2525-3409 | DOI: http://dx.doi.org/10.33448/rsd-v10i14.22343

\section{Continuação da Tabela 1}

Peretti-Watel et al., 2019

França

Pais e/ou responsáveis

Alto nível

alunos de 3 a 5 anos, das

creches selecionadas

- Medo dos efeitos;

- Associa algumas

- Internet como fonte de informação; doenças como

consequência de

vacinas;

Danjuma et al., 2020

Nigéria

Mães de recém-nascidos

Nível intermediário

- $\quad$ Prematuridade com

baixo peso ao nascer;

- Parto em hospitais

privado, as vacinas

devem ser agendadas.

- Parto em hospital privado expressa adm.

Tardia de vacinas.

Fonte: Autores. 


\section{Discussão}

Diante dos resultados obtidos constatou-se que existem motivos para os pais e/ou responsáveis não realizarem as vacinas das crianças de 0 a 5 anos, os principais motivos relatados foram: julgar as vacinas como sendo desnecessárias, medo dos efeitos colaterais e optar por estilos de vida mais naturais. Esses relatos são observados em estudos propostos no Brasil, França, Estados Unidos, Canadá e Austrália, nos últimos 5 anos.

Além dos motivos para não realizar as vacinas ainda existem os fatores que irão interferir nesse processo, sendo mais uma barreira a ser vencida por parte da equipe de saúde pública. Sendo a ausência de imunobiológicos e a cultura familiar os principais (Barros et al., 2015).

Dentro do dimensionamento sociocultural do acesso, “A não vacinação está relacionada ao medo da dor, à falta de conhecimento, aos costumes e crenças que a sociedade traz juntamente com seus valores culturais”. Cada indivíduo tem liberdade de expressão e de decisão sobre si, carregando motivos sociais, culturais e religiosos que influem nesta decisão (Ferreira et al., 2017).

Hoje, não é muito adequado usar o fator de escolaridade como obstáculo para cumprir esse calendário, pois observamos que a maioria das pessoas que aderem ao ato de não vacinar seus filhos são aqueles que têm uma boa escolaridade e possuem argumentos para justificar suas atitudes, abordando que existe outras maneiras de prevenir, e que as vacinas causam reações adversas e isso não é bom, há também os pais autoritários que não quer opinião sobre o seu agir, estes então deixam de garantir uma proteção ao seu filho (Barbieri \& Couto \& Aith, 2017).

Em pesquisa realizada no Canadá a dificuldade para ter acesso a vacina era observada na minoria dos indivíduos, entretanto essa população era formada por pais que tinham baixa, nenhuma ou moderada intenção de realizar a vacina nos seus filhos. Avaliando o conhecimento desses pais 67,8\% deles consideraram que o seu nível de conhecimento sobre vacinação era bom, verificando que o problema para um decréscimo no nível de cobertura vacinal, não é a falta de conhecimento (Dubé et al., 2018).

Diferentes estudos são contra a vacina da hepatite B, os questionamentos encontrados acerca de seus efeitos colaterais são vários inter telespectadores mencionaram efeitos colaterais potenciais muito sérios, como esclerose múltipla por hepatite B, outros abordam que a hepatite B é uma doença de adultos, portanto, seus filhos devem decidir por si próprios vacinar-se ou não quando forem adultos (Peretti-Watel et al., 2019). A maioria dos participantes deste estudo tinham seus conhecimentos adquiridos através da internet, sendo está um obstáculo a ser enfrentado pelo Programa Nacional de Imunização com informações irrelevantes e o chamado "Fake News".

Mediante observação dos resultados, chama atenção o ato de não vacinar estar relacionado a crenças, em algumas regiões fatores religiosos irão ter forte impacto na redução da cobertura vacinal, estudos mostraram que os membros do grupo kavonokya no condado de Kitui, relataram evitar a utilização do serviço de imunização (Nzioki et al., 2017). As crenças estão inseridas nos fatores que impedem a vacinação, apesar de nenhum estudo ter justificativa mais profunda para essa prática.

Em estudo no Canadá quase metade dos participantes (47,8\%) relataram que seus familiares tinham influências de práticas religiosas ou espirituais que interferiam nas suas escolhas de saúde, como comparado aos pais com nenhuma, baixa ou moderada intenção de vacinar, aqueles que tinham forte intenção de vacinar eram menos propensos a afirmar que as práticas religiosas ou espirituais influenciavam suas escolhas de saúde (Dubé et al., 2018). Evidenciando que os pais com altas intenções de vacinar não usavam a crença como obstáculo.

Em países como Estados Unidos, o custo das vacinas foram fatores que limitaram as oportunidades de absorção das mesmas, na Austrália menos de 3/4 dos entrevistados relataram que podem pagar a vacina contra hepatite A, brancos também eram mais propensos a pagar a vacina do que os não-brancos, como eram indivíduos casados ou comprometidos, e indivíduos 
com ensino superior e maior renda. Aqueles que não podiam pagar pela vacina, metade indicou que seriam imunizados se a vacina fosse oferecida gratuitamente (Biezen et al., 2018).

É notório o reaparecimento em alguns países de doenças que já haviam sido erradicadas, esse acontecido se dá devido aos pais que recusam vacinas, os mesmos citam temor de que vacinas pode ter efeitos colaterais prejudiciais (Horne et al., 2015). O acesso à internet traz muitos malefícios, pois há várias informações falso-positivas que influenciam de forma negativa no pensamento dos pais, muitos até possuem dúvidas das informações encontradas (Peretti-Watel et al., 2019). Não vacinar as crianças como decisão dos pais, tem despertado grande curiosidade aos pesquisadores de diversas áreas, que tentam entender o porquê de tal ato, tendo uma hipótese da relação entre o contexto social do indivíduo e numerosas concepções de risco (Medeiros, 2019).

Muitos pais não têm conhecimentos sobre o contexto das vacinas mais realizam o ato, já que existe a vacina obrigatória e gratuita disponível, a tal chamada "cultura da imunização", outros conhecem a eficácia e confiabilidade dos imunobiológicos, sabe-se o seu papel e o direito da criança em prol da saúde e contribuem positivamente no cumprimento do cartão (Barbieri \& Couto \& Aith, 2017).

Dentro do sistema ainda existem as falhas relatadas pelos pais e/ou responsáveis relacionada aos profissionais de saúde muitos não conhecem os efeitos adversos de algumas vacinas, deixando assim de orientar os responsáveis, também houve constatação de situações de falsa contraindicação, em que os vacinadores decidiram não realizar a vacinação embora não houvesse, de fato, contraindicação do procedimento. A educação em saúde é uma importante ferramenta da atenção básica, a melhoria da qualidade do processo de comunicação entre o serviço de saúde e as famílias, valorizada pela educação continuada dos profissionais, é fundamental para minimizar as perdas de oportunidade de vacinação (Barros et al., 2015).

A cerca do entendimento dos profissionais pela vacina, é necessário um melhor treinamento destes, através da educação continuada, muitos não conhecem a base dos imunobiológicos que estão sendo ofertados, abordam que o calendário sofre alterações que os deixam em dúvidas no momento assistencial, além de existir dificuldade em realizar a técnica correta de administração. Em pesquisa realizada observou que há uma centralização dos relatórios das atividades de vacinas apenas a secretária de saúde, os trabalhadores não têm o conhecimento dos indicadores de seu território, do percentual de cobertura alcançada das taxas de abandono que ocorre em seu espaço, não podendo intervir para melhorar aquela realidade (Aragão et al., 2019).

Entrega de fim de semana, entrega fora dos dias de vacinação de rotina e em feriados, falta de estoque de vacinas e prematuridade com baixo peso ao nascer eram todas as barreiras para a administração oportuna de vacinas, levando assim a imunização tardia das vacinas que eram obrigatórias ao nascer, o parto em hospital privado foi o único fator que significativamente justificou a administração tardia de vacinas no nascimento, pelo fato de serem agendadas a alta demanda faz com que a espera seja maior que o prazo previsto (Danjuma et al., 2020).

Um importante fator que acaba impedindo o cumprimento do calendário de crianças menos de 5 anos, é a falta de conhecimento dos profissionais de saúde, alguns atrasam as vacinas por motivos de falsas contraindicações, em relação a vacina da hepatite $\mathrm{B}$, frente ao parto domiciliar esses profissionais não levaram as vacinas até essas crianças, deixando a oportunidade passar, sabemos que a vacina contra hepatite B deve ser administrada dentro de 24 horas após o nascimento, e os profissionais "desinformados" não levam isso em consideração (Li et al., 2017).

Observa-se também em entrevista realizada com profissionais no Brasil, determinado percentual não conheciam os eventos adversos das vacinas, nem os tipos de vacinas que tinha naquele local, também ocorreu falsas contraindicações (Barros et al., 2015). Diante dessas perspectivas muitos pais se tornam mais rígidos para não realizar a vacinação de seus pequenos. Ocorreu relato de erros por parte do vacinador levando a uma grande problemática para o serviço de saúde, o profissional e até mesmo a crianças (Dubé et al., 2018). 
Os pais e/ou responsáveis tem a guarda de seus filhos e decidem o que fazer com eles, mas quando o mesmo se contrapõe ao que é bom para a crianças, sua atitude passa a ser interrogada, são vários os motivos que levam estes, a não imunizar seus menores, em contrapartida existe uma lei que os obriga a realizar o ato, para que possa proteger a sua criança e as demais, excluindo todos os relatos falsos e buscando fontes verdadeiras e confiáveis de informação. Uma série de doenças conseguirão ser erradicadas no país, por meio da imunização como forma de prevenção de novos casos e ações de promoção à saúde ao orientar as pessoas a vacinarem seus filhos.

Com a criação do PNI as vacinas passaram a ser integradas no calendário de vacinação, proposto anualmente pelo Ministério da Saúde, de acordo com o surgimento de novos imunizantes para determinadas doenças. "Outro ponto em questão que merece destaque é o calendário de vacinação, que em seu início era composto apenas contra sete doenças, e foi se expandindo gradativamente" (Lima et al.,2017), essa expansão se deu pela eficácia dos imunobiológicos, que por sua vez reduziu o quadro de morbimortalidade infantil no país.

O Brasil e o Mundo têm eficácia e comprovação da vacinação, hoje em 2020 nos encontramos frente a pandemia da COVID-19, pesquisadores do mundo todo estão em estudos para desenvolver uma vacina capaz de combater o vírus, porque essa é a medida mais eficaz de controle de uma doença, o diretor-geral da Organização Mundial de Saúde (OMS), Tedros Adhanom Ghebreyesus, disse que, "enquanto o mundo confronta a pandemia de COVID-19, a vitória da humanidade sobre a varíola é um lembrete do que é possível quando as nações se reúnem para combater uma ameaça comum à saúde" (OPAS/OMS, 2020).

\section{Considerações Finais}

A vacina é a fonte mais eficaz de proteção ao indivíduo, ela garante imunização acerca de diversas doenças, tornando o mesmo imune a vários microrganismos, durante décadas ela vem sendo usada por diversos países no mundo, sendo comprovada sua positividade na vida das pessoas, e sendo o motivo de erradicação de diversas patologias potencialmente fatais. Este trabalho teve como problemática o motivo de pais e/ou responsáveis de crianças de 0 a 5 anos não cumprirem o calendário vacinal.

Diante disso os resultados encontrados nesta pesquisa como as barreiras citadas por pais e/ou responsáveis, é algo que pode ser solucionado por parte dos mesmos e do serviço de saúde.

Foi notado que existem um quantitativo de pais em diversos países, que escolhem por não vacinar seus filhos, estes devem abster-se de fonte inconfiáveis de informação, como a internet, sites e grupos que tentam impor o chamado "movimento antivacinas", pois este traz informações incoerentes, opostas com aquelas cientificamente comprovada. Dessa maneira, os pais e/ou responsáveis que abordaram achar desnecessárias ou inseguras as vacinas, ter dúvidas sobre sua eficácia, provavelmente se apegam a fontes incompletas de informação, sendo isso uma barreira a ser solucionada.

Por outro lado, o serviço público de saúde precisa melhorar em alguns pontos, como no fornecimento de imunobiológicos para os serviços de atenção básica, fazendo com que os estoques se mantenham abastecidos para não haver falta dos mesmos. Ampliar a educação permanente dos profissionais que atuam no setor de vacina, melhorando seus conhecimentos e técnicas; executar palestras com a sociedade sobre o tema "vacinação e seus benefícios", tirando as dúvidas que os indivíduos tiverem sobre isso, os agentes de saúde também, devem realizar a busca das crianças com vacinas atrasadas e verificar as barreiras para isso, podendo incentivar esses pais a tirarem dúvidas e perder seu temor acerca das vacinas.

O fator mais aceitável nesse estudo que pode ser encarado como uma justificativa para não vacinar, e deve ser respeitado são aqueles pais ou responsáveis que optam pelo estilo de vida natural para a vida de seus filhos, sendo uma cultura familiar adotada pela família que não pode ser modificado, também respeitando os demais motivos que foram abordados neste 
estudo. Concluindo que cada pessoa tem o direito de decidir sobre si, mas não esquecendo que existem leis para guiar nossas ações e nos ajudar em como agir diante de determinadas situações na vida.

\section{Referências}

Aragão, R. F., Napoleão, A., I. M., Ribeiro, M. A., Barreto, R. M., \& Sousa, J. A. (2019). Percepções e conhecimentos da equipe de enfermagem sobre o processo de imunização. Revista Brasileira Em Promoção da Saúde, 32, 1-8. https://doi.org/10.5020/18061230.2019.8809

Barbieri, C. L. A., Couto, M. T. \& Aith, F. M. A. (2017, 9 de março). A (não) vacinação infantil entre a cultura e a lei: os significados atribuídos por casais de camadas médias de São Paulo, Brasil. Cadernos de Saúde Pública [online]. 2017, v. 33, n. 2 https://doi.org/10.1590/0102-311X00173315

Barros, M. G. M. et al. (2015). Perda de oportunidade de vacinação: aspectos relacionados à atuação da atenção primária em Recife, Pernambuco, 2012. Epidemiologia e Serviços de Saúde [online]. v. 24, n. 4. https://doi.org/10.5123/S1679-49742015000400012

Biezen, R., Grando, D., Mazza, D., \& Brijnath, B. (2018). Why do we not want to recommend influenza vaccination to young children? A qualitative study of Australian parents and primary care providers. Vaccine, 36(6), 859-865. https://doi.org/10.1016/j.vaccine.2017.12.066

Braz, R. M., Domingues, C. M. A. S., Teixeira, A. M. da S., \& Luna, E. J. de A. (2016). Classificação de risco de transmissão de doenças imunopreveníveis a partir de indicadores de coberturas vacinais nos municípios brasileiros. Epidemiologia e Servicos de Saude: Revista Do Sistema Unico de Saude Do Brasil, 25(4), 745-754. https://doi.org/10.5123/S1679-49742016000400008

Civil, C. (2020). Presidência da República. 2-4. Decreto $\mathrm{n}^{\circ}$ : 78.231/76. http://www.planalto.gov.br/ccivil_03/decreto/19701979/d78231.htm\#: :text=DECRETO\%20No\%2078.231\%2C\%20DE,doen\%C3\%A7as\%2C\%20e\%20d\%C3\%A1\%20outras\%20provid\%C3\%AAncias.

Clara, M., Araújo, G., Silva, L. F., Baldoino, L. S., Naiana, T., Porto, R. S., Martins, V. D. S., \& Carvalho, D. P. (n.d.). Fatores que interferem no cumprimento do calendário vacinal na infância Factors that interfere with the fulfillment of the childhood immunization schedule Factores que interfieren en el cumplimiento del calendario de vacunación en la infância. 1-10.

Danjuma, S. Ibrahim, A. Shehu, N. Diala, M. Pam, C. Ogbodo, C. (2020). Oportunidade de vacinação ao nascimento: Uma análise de inatos nas terras altas de Jos, centro-norte da Nigéria.

Dubé, E., Gagnon, D., Ouakki, M., Bettinger, J. A., Witteman, H. O., MacDonald, S., Fisher, W., Saini, V., \& Greyson, D. (2018). Measuring vaccine acceptance among Canadian parents: A survey of the Canadian Immunization Research Network. Vaccine, 36(4), 545-552. https://doi.org/10.1016/j.vaccine.2017.12.005

Ferreira, A. V., Oliveira, C. F., Guimarães, E. A. de A., Cavalcante, R. B., Moraes, J. T., \& Oliveira, V. C. de. (2017). Acesso à sala de vacinas nos serviços de atenção primária à saúde. Revista Eletrônica de Enfermagem, 19(1), 1-11. https://doi.org/10.5216/ree.v19.42468

Ferreira, V. L. de R., Waldman, E. A., Rodrigues, L. C., Martineli, E., Costa, Â. A., Inenami, M., \& Sato, A. P. S. (2018). Assessment of vaccination coverage of children in a medium-sized brazilian city using electronic immunization registry. Cadernos de Saude Publica, 34(9). https://doi.org/10.1590/0102$311 \times 00184317$

Filgueiras, R. D. S., Liz, M., Schaustz, R., \& Fonseca, S. C. (2018). eletrônico no município do Rio de Janeiro Vaccinal coverage in children until up to two years in a Municipal Health Center in Rio de Janeiro. 39-45. https://doi.org/10.24118/reva1806.9495.3.1.2018.402

Horne, Z., Powell, D., Hummel, J. E., \& Holyoak, K. J. (2015). Countering antivaccination attitudes. Proceedings of the National Academy of Sciences of the United States of America, 112(33), 10321-10324. https://doi.org/10.1073/pnas.1504019112

Lima, A. A., \& Pinto, E. D. S. (2017). O contexto histórico da implantação do Programa Nacional de Imunização (PNI) e sua importância para o Sistema Único de Saúde (SUS). Scire Salutis, 7(1), 53-62. https://doi.org/10.6008/spc2236-9600.2017.001.0005

Medeiros, M. F. (2019). Os Movimentos Contra Vacinação No Brasil E a Lei Da Vacinação Obrigatória: Uma Análise Crítica a Partir Dos Direitos Da Criança E Do Adolescente E a Partir Do Risco De Surtos Epidêmicos De Doenças Infecciosas Anteriormente Controladas Por Cobertura Vacina. Revista Dissertar, 1(32), 93-106. https://doi.org/10.24119/16760867ed115272

Mizuta, A. H., Succi, G. de M., Montalli, V. A. M., \& Succi, R. C. de M. (2018). Percepções Acerca Da Importância Das Vacinas E Da Recusa Vacinal Numa Escola De Medicina Tt - Perceptions on the Importance of Vaccination and Vaccine Refusal in a Medical School. Revista Paulista de Pediatria, 37(1), 34-40. https://doi.org/10.1590/1984-0462/;2019;37;1;00008

Nzioki, J. M., Ouma, J., Ombaka, J. H., \& Onyango, R. O. (2017). Community health worker interventions are key to optimal infant immunization coverage, evidence from a pretest-posttest experiment in Mwingi, Kenya. Pan African Medical Journal, 28, 1-13. https://doi.org/10.11604/pamj.2017.28.21.11255

Peretti-Watel, P., Ward, J. K., Vergelys, C., Bocquier, A., Raude, J., \& Verger, P. (2019). 'I Think I Made The Right Decision ... I Hope I'm Not Wrong'. Vaccine hesitancy, commitment and trust among parents of young children. Sociology of Health and Illness, 41(6), 1192-1206. https://doi.org/10.1111/14679566.12902

Repa, T. P, Silva, D.R. da. (2018). A Relevância da Cobertura Vacinal e da Imunização em Crianças no Brasil. Centro Universitário FAG- Cascavel, Paraná, Brasil. 2018. http://tcconline.fag.edu.br:8080/app/webroot/files/trabalhos/20181210-213212.pdf

Silva, F. de S., Barbosa, Y. C., Batalha, M. A., Ribeiro, M. R. C., Simões, V. M. F., Branco, M. D. R. F. C., Thomaz, É. B. A. F., Queiroz, R. C. de S., Araújo, W. R. M., \& Da Silva, A. A. M. (2018). Incompletude vacinal infantil de vacinas novas e antigas e fatores associados: Coorte de nascimento BRISA, São Luís, Maranhão, Nordeste do Brasil. Cadernos de Saude Publica, 34(3). https://doi.org/10.1590/0102-311x00041717 
Research, Society and Development, v. 10, n. 14, e531101422343, 2021

(CC BY 4.0) | ISSN 2525-3409 | DOI: http://dx.doi.org/10.33448/rsd-v10i14.22343

Soares, C. B. (2010). Revisão Integrativa versus Revisão Sistemática. Reme: Revista Mineira de Enfermagem, v. 8, n. 1, p. 102-10. 6, 2010. https://doi.org/10.1590/S1679-45082010RW1134

Tedros Adhanom Ghebreyesus. (2020). Erradicação da varíola: um legado de esperança para COVID-19 e outras doenças. https://www.paho.org/bra/index.php?option=com_content\&view=article\&id=6165:erradicacao-da-variola-um-legado-de-esperanca-para-covid-19-e-outrasdoencas\&Itemid=812.

$\mathrm{Xi}$, L., (2017). Improving hepatites $\mathrm{B}$ birth dose coverage through village health volunteer training and pregnant women education. https://doi.org/10.1016/j.vaccine.2017.06.056 